La Revue

des Droits

de l'Homme

\section{La Revue des droits de l'homme}

Revue du Centre de recherches et d'études sur les droits fondamentaux

$4 \mid 2013$

Revue des droits de l'homme - $\mathrm{N}^{\circ} 4$

\title{
Entretien avec Jean-Marie Delarue
}

Contrôleur général des lieux de privation des libertés

Véronique Champeil-Desplats et Catherine Teitgen-Colly

\section{(2) OpenEdition}

\section{Journals}

Édition électronique

URL : http://journals.openedition.org/revdh/403

DOI : $10.4000 /$ revdh.403

ISSN : 2264-119X

Éditeur

Centre de recherches et d'études sur les droits fondamentaux

Référence électronique

Véronique Champeil-Desplats et Catherine Teitgen-Colly, « Entretien avec Jean-Marie Delarue », La Revue des droits de l'homme [En ligne], 4 | 2013, mis en ligne le 01 décembre 2013, consulté le 10 juillet 2020. URL : http://journals.openedition.org/revdh/403 ; DOI : https://doi.org/10.4000/revdh.403

Ce document a été généré automatiquement le 10 juillet 2020

Tous droits réservés 


\title{
Entretien avec Jean-Marie Delarue
}

\author{
Contrôleur général des lieux de privation des libertés \\ Véronique Champeil-Desplats et Catherine Teitgen-Colly
}

\begin{abstract}
Le Contrôleur général des lieux de privation des libertés (CGLPL) a pour mission de veiller au respect des droits fondamentaux des personnes privés de liberté. Comment concevezvous cette mission?

Beaucoup se sont interrogés sur la signification de l'expression «droits fondamentaux ». Certains en ont proposé une conception restrictive: les droits fondamentaux ne désigneraient qu'un noyau dur. Cette étiquette de «fondamental » pourrait les desservir! Il existerait, par conséquent, au sein des droits de l'Homme des droits plus importants que d'autres. Je ne partage pas cette lecture. Les droits fondamentaux doivent être compris comme ceux nécessaires à la vie quotidienne. Il faut les envisager, comme invite d'ailleurs à le faire la Cour européenne des droits de l'Homme, de façon pragmatique, concrète, dans les détails de la quotidienneté : contrôle de l'exhaustivité et de la précision des registres des autorités pénitentiaires ou de police, appréciation de la régularité des rondes, de l'intensité des fouilles, de l'information délivrée aux personnes privées de liberté, de la capacité de celles-ci à correspondre avec l'extérieur dans le respect du secret de leurs correspondances, des moyens ordinaires mis à disposition pour se défendre contre une mesure ou pour exercer la liberté d'expression, pour écrire...

La mission de protection des droits fondamentaux exercée par le CGLPL se veut par conséquent extrêmement concrète. Elle scrute les détails à la manière d'un ethnologue. Il ne sert en effet à rien d'affirmer que les personnes privées de liberté ont des droits fondamentaux si ces droits ne sont pas effectifs, s'il est fait obstacle à leur exercice au jour le jour, ici en refusant de fournir des stylos, là en enfermant deux ou trois personnes dans une cellule de $8 \mathrm{~m}^{2}$. La description froide, objective et détaillée qui peut être observé au cours de nos visites est souvent suffisante pour mettre à jour les violations quotidiennes des droits.
\end{abstract}

Comment vos actions s'articulent-elles avec celles des juges nationaux?

Les rapports avec les juridictions internes se construisent progressivement. Le CGLPL produit des avis et des rapports qui sont mis à la disposition de toute institution 
publique, y compris les juges. Quelques tribunaux administratifs, à l'occasion de référés-constat, ont commencé à citer des avis et des recommandations que nous avions rendus. Cela a été le cas tout particulièrement de la recommandation relative au centre pénitentiaire des Baumettes à Marseille. On peut aussi évoquer des références dans les visas d'un jugement de Tribunal administratif de notre avis sur la pratique du culte dans les lieux privatifs de liberté. Nous souhaiterions que ces initiatives se développent comme cela est le cas devant la Cour de l'Européenne des droits de l'Homme. Celle-ci n'hésite plus, depuis longtemps, à prendre appui sur les rapports du Comité européen pour la prévention de la torture et des peines ou traitements inhumains ou dégradants. La Cour a même cité à quelques reprises les observations du CGLPL.

De telles références présentent selon nous l'intérêt de contribuer à « objectiver » et à généraliser les cas contentieux pour tirer des conclusions touchant à l'organisation même du service public et à son fonctionnement. Elles signifient, qu'au-delà du recours individuel, la situation contestée concerne structurellement l'ensemble d'un établissement ou d'un type d'établissements de privation de la liberté. En outre, les informations mises à la disposition du juge après enquête de terrain effectuée par nos agents contribuent à établir une certaine égalité entre l'administration et la personne privée de liberté qui est souvent dans l'incapacité de fournir des preuves. Plus le CGLPL développera donc ses champs d'investigation, plus les individus privés de liberté disposeront de ressources pour se défendre, et plus les chances de voir évoluer des jurisprudences seront importantes.

Car, si sous l'influence de la Cour européenne des droits de l'homme, le Conseil d'Etat a déjà parcouru depuis le milieu des années 1990 un chemin important, il reste encore de nombreux points à améliorer que nos rapports ou avis n'ont pas hésité à mettre en exergue. L'abandon, sauf exception dûment justifiée, de l'emploi des visioconférences en audience en fournit un exemple. La Cour européenne n'est ellemême pas allée aussi loin que nous le pensons possible.

Un processus dialectique entre la Cour européenne des droits de l'Homme, les juges nationaux et le CGLPL s'engage néanmoins et nous souhaiterions vivement qu'il se développe davantage afin de renforcer la dynamique de protection et d'effectivité des droits.

Puisque vous évoquez la Cour européenne, quels liens le CGLPL entretient-il avec les institutions européennes et internationales intervenant dans son domaine?

Comme nous l'avons signalé la Cour européenne n'hésite pas à se référer à nos avis et rapports, même si en 2011 dans l'affaire Stasi contre France sur la protection d'un détenu à l'égard de ses co-détenus, elle n'a pas condamné la France alors que nous avions des éléments portant à conclure à une solution contraire.

Nous entretenons également de très bonnes relations avec le Comité européen pour la prévention de la torture et des peines ou traitements inhumains ou dégradants. Nous avons collaboré à chacune des visites que celui-ci a effectuées en France. L'articulation est moins aisée avec l'Union européenne. Reste notamment en jachère le chantier qui avait été ouvert sur la détermination de règles relatives à la rétention administrative des étrangers. Nous nous sommes aussi rapprochés du Sous-comité contre la Torture des Nations-Unies. 
Ces collaborations et contacts internationaux sont indispensables. Nous nous sommes également engagés dans des opérations bilatérales de coopération avec des institutions de prévention d'autres Etats, la République tchèque, le Royaume-Uni tout particulièrement.

Pour en revenir au niveau national, quelles relations entretenez-vous avec les autres autorités administratives indépendantes (AAI) ? Comment concevez-vous la répartition de vos compétences avec celle du Défenseur des droits qui a récemment publié un rapport sur son action auprès des personnes détenues?

Nous avons signé avec certaines AAI des conventions de collaboration sur des sujets où nos compétences s'entrecroisent. C'est le cas avec le Conseil supérieur de l'audiovisuel sur le droit à l'image des détenus ou avec la Commission nationale informatique et liberté sur les fichiers de police par exemple. Mais, dans tous les cas, ces collaborations s'effectuent avec la préoccupation majeure de maintenir l'indépendance de chaque institution. Le CGLPL ne reçoit aucune consigne de quiconque et reste libre d'utiliser et de réapprécier les informations qui lui sont fournies.

Quant au Défenseur des droits, dont l'institution s'inscrit dans la prolongation du Médiateur de la République, il exerce essentiellement une compétence de médiation. Il résout des conflits entre l'administré et l'administration à la demande du premier qui n'ont pu l'être par d'autres voies. Le CGLPL exerce pour sa part la fonction de prévention qu'exige le protocole qui le fonde, le protocole facultatif se rapportant à la Convention contre la torture et autres peines et traitements cruels, inhumains et dégradants adopté par l'Assemblée générale des Nations-Unies le 18 décembre 2002. Autrement dit, l'un a un rôle curatif, affaire par affaire, d'où peuvent se déduire des questions générales; l'autre un rôle préventif ( $2 / 3$ des visites des lieux de privation de liberté ne sont pas programmées) et principalement axé sur le fonctionnement et l'organisation des lieux. Le CGLPL se concentre sur la question de savoir si oui ou non l'Etat se dote des mécanismes nécessaires et suffisants pour lutter contre la torture et les traitements inhumains et dégradants dans les lieux où des individus sont privés de liberté.

On perçoit ici le risque d'une éventuelle fusion des institutions, à savoir l'absorption du rôle préventif par la fonction curative. Or, le Conseil de l'Europe a plusieurs fois souligné la nécessité de soutenir des politiques publiques de prévention. Même, dans les pays européens où il existe traditionnellement une autorité centralisée de garantie des droits face à l'administration, alors également chargée de la question des lieux privatifs de liberté (le Defensor del Pueblo en Espagne, les Ombudsmans dans les pays scandinaves), la double perspective de prévention et de résolution des conflits reste bien séparée dans les institutions, sous peine d'être contre-productive.

On peut reconnaitre qu'aujourd'hui, institutionnellement, la France se trouve dans une situation exemplaire quant à l'importance donnée au contrôle des lieux privatifs de libertés. Et le travail ne manque pas. Il ne faut donc pas affaiblir les dispositifs créés. Il y a bien place pour deux institutions exerçant chacun des fonctions différentes et complémentaires. A ce titre, pour éviter des confusions quant à la répartition des compétences qui pourraient être préjudiciable aux requérants, j'ai demandé au Défenseur des droits, comme nous y avons procédé avec d'autres autorités administratives indépendantes, de signer une convention qui facilite la transmission du courrier et des informations sur les dossiers. Elle a été signée dès 
2011. Il s'agit de collaborer au mieux tout particulièrement lorsqu'une requête présente des questions qui ressortent de la compétence à la fois du Défenseur des droits et du CGLPL. Il en va de l'intérêt des personnes à protéger. 